\title{
Athena: Uma Ferramenta Visual para Auxiliar o Ensino da Inteligência Computacional
}

\author{
Ronyérison Braga $^{1}$, Pedro Almir Oliveira ${ }^{2}$, Matheus Souza ${ }^{1}$, \\ Ricardo Britto $^{3}$, Ricardo Rabêlo ${ }^{1}$, Pedro Santos Neto ${ }^{1}$ \\ ${ }^{1}$ Universidade Federal do Piauí \\ Teresina - Piauí - Brasil \\ ${ }^{2}$ Instituto Federal do Maranhão \\ Pedreiras - Maranhão - Brasil \\ ${ }^{3}$ Blekinge Institute of Technology \\ Karlskrona - Sweden \\ ronyerisonbraga@gmail.com, pedro.oliveira@ifma.edu.br, matheusmmcs@gmail.com \\ ricardo.britto@bth.se, \{ricardoalr, pasn\}@ufpi.edu.br
}

\begin{abstract}
Computational Intelligence (CI) techniques have been used in many different knowledge areas in order to rationalize costs and potentially increase profits. Nevertheless, using this kind of technique is not straight forward, especially in the case of sectors whose practitioners do not have enough programming knowledge. In order to facilitate the teaching, learning, research and usage of CI techniques, we have developed a tool called Athena. In this paper, we report the results of an experiment that we conducted to identify to which extent Athena improves the performance of students during the learning of IC techniques. The results indicate that our tool can be very useful for educators involved in teaching IC.
\end{abstract}

Resumo. Técnicas de Inteligência Computacional (IC) têm sido utilizadas amplamente nas mais diversas áreas de conhecimento, de modo a racionalizar custos e potencialmente aumentar lucros. Porém, usar esse tipo de técnica não é trivial, especialmente em setores cujos profissionais não tem grande conhecimento de programação. Visando facilitar o ensino, aprendizado, pesquisa e o uso de técnicas de IC, desenvolveu-se a Athena. Neste artigo, são reportados os resultados de um experimento que foi conduzido para identificar até que ponto o uso dessa ferramenta melhora o desempenho de alunos durante o aprendizado de técnicas de IC. Os resultados indicam que a Athena pode ser útil para educadores envolvidos com o ensino de IC.

\section{Introdução}

A Inteligência Computacional é uma subárea da Inteligência Artificial (IA) concentrada no estudo de mecanismos adaptativos para possibilitar ou facilitar o comportamento inteligente de sistemas em ambientes complexos e dinâmicos. Essa área reúne diferentes técnicas que exploram a tolerância à precisão, incerteza e verdades parciais para obter flexibilidade, robustez, soluções de baixo custo e boa adequação em problemas reais [Engelbrecht 2007]. 
V Congresso Brasileiro de Informática na Educação (CBIE 2016)

Anais do XXVII Simpósio Brasileiro de Informática na Educação (SBIE 2016)

As técnicas de IC vêm obtendo bastante sucesso na resolução de problemascomplexos nas mais diversas áreas do conhecimento, por exemplo, no ensino de programação [Azevedo and Tavares 1998], no reconhecimento de distúrbios na qualidade de energia elétrica [Zhu et al. 2004], na priorização de casos de teste em desenvolvimento de software [Santos-Neto et al. 2012], em problemas de alocação de equipes [Britto et al. 2012], em diagnósticos médicos [Amato et al. 2013], aplicações financeiras [Diekmann and Gutjahr 1998], dentre outros.

Entretanto, apesar do grande número de aplicações para a IC, poucos profissionais utilizam as técnicas de IC com frequência, provavelmente devido à falta de instrução - normalmente é necessário ter um conhecimento robusto de programação - ou pelo alto custo de desenvolvimento. Dessa forma, desenvolveu-se a ferramenta Athena para apoiar pesquisadores no desenvolvimento de Sistemas Inteligentes [Oliveira et al. 2014]. Contudo, após sua utilização por alguns grupos de pesquisa, percebeu-se que tal ferramenta poderia ser utilizada por professores e alunos no ensino e na aprendizagem das técnicas de IC, principalmente devido à disponibilização de um editor gráfico que facilita a construção, manutenção e aplicação de Sistemas Inteligentes (que utilizam técnicas de IC).

Este trabalho tem por objetivo apresentar a ferramenta Athena, desenvolvida para aprimorar o apoio à construção de Sistemas Inteligentes e que pode ser utilizada no ensino das técnicas de IC. Dessa forma, as principais contribuições do trabalho são:

- Apresentar a ferramenta Athena que pode auxiliar o ensino da IC. Ela oferece um ambiente visual no qual os professores podem apresentar o funcionamento das principais técnicas de IC durante as aulas, gerando uma maior integração entre a teoria e a prática;

- Possibilitar o ensino da IC nas mais diversas áreas de conhecimento, uma vez que, com a utilização da Athena, o usuário poderá construir sistemas de IC sem a necessidade de conhecimentos em linguagens de programação;

- Discutir um estudo experimental realizado em ambiente controlado com o objetivo de avaliar o desempenho de alunos utilizando a ferramenta na construção de Sistemas Inteligentes para a resolução de problemas reais.

O restante deste trabalho está organizado da seguinte forma: a Seção 2 apresenta os principais trabalhos relacionados à proposta da Athena e que possuem relação com o ensino; a Seção 3 descreve a ferramenta proposta; na Seção 4 são apresentados os resultados do estudo experimental realizado com alunos de graduação e pós-graduação; e, finalmente, a Seção 5 apresenta as conclusões e perspectivas para trabalhos futuros.

\section{Trabalhos Relacionados}

De modo a identificar trabalhos que propõem ferramentas para apoiar aplicação, ensino e aprendizado de IC, foi realizado um Mapeamento Sistemático da Literatura relacionado a essa questão [Braga et al. 2015]. Como resultado, foram identificados 24 artigos relevantes. Porém, o foco dos trabalhos encontrados está mais relacionado no apoio a aplicação de técnicas de IC. Nenhum trabalho incluído no mapeamento foca no ensino e aprendizado de IC. Isso ressalta a importância deste estudo, que propõe uma ferramenta capaz de auxiliar o ensino das técnicas de IC.

Existem trabalhos que propõem ferramentas com a capacidade de apoiar o ensino e aprendizado de Engenharia de Software. Por exemplo, Laroza e Seabra 
V Congresso Brasileiro de Informática na Educação (CBIE 2016)

Anais do XXVII Simpósio Brasileiro de Informática na Educação (SBIE 2016)

[Laroza and Seabra 2015] propuseram uma abordagem para apoiar o ensino da linguagem UML, por meio de uma ferramenta educacional denominada REA-UML. Durante a realização do trabalho foi realizado um estudo experimental no qual os pesquisadores, inicialmente, ofereceram aulas a alunos do curso de Ciência da Computação de uma universidade sobre modelagem de sistemas orientados a objetos por meio da linguagem UML e ao final do período de utilização da ferramenta os alunos responderam a um questionário com o propósito de avaliar a percepção de qualidade dos participantes a respeito da ferramenta REA-UML.

Monteiro et al. [Monteiro et al. 2015] propuseram um ambiente para apoiar a aprendizagem ubíqua sob uma licença opensource. O projeto disponibiliza um cliente adaptado à plataforma mobile para tablets e smartphones com sistema operacional Android. Os pesquisadores realizaram uma avaliação do projeto com professores e alunos e obtiveram como resultados indícios de que houve aumento no engajamento de aprendizes entre os participantes que utilizaram o ambiente proposto.

\section{A Ferramenta Athena}

Atualmente, quando um aluno, pesquisador ou profissional deseja empregar um técnica de Inteligência Computacional para resolver determinado problema, destacam-se quatro tarefas importantes: i) modelar o problema-alvo; ii) desenvolver uma aplicação utilizando ferramentas especializadas; iii) realizar experimentos para avaliar os resultados obtidos; e iv) integrar a aplicação com outros sistemas.

A primeira tarefa requer conhecimentos sobre o problema-alvo e sobre as técnicas escolhidas para solucioná-lo. A segunda tarefa exige conhecimento teórico na ferramenta utilizada para apoiar o desenvolvimento. A maioria dessas ferramentas exige conhecimentos em linguagens de programação para viabilizar a construção da aplicação. A terceira tarefa é importante para ajustar os parâmetros das técnicas e, por fim, a quarta tarefa é responsável por integrar o que foi desenvolvido com outros sistemas. A realização dessas tarefas envolve a superação de uma série de desafios. Dessa forma, a Athena ${ }^{1}$ foi desenvolvida para facilitar a superação dessas desafios, possibilitando construção, manutenção e aplicação de Sistemas de Inteligência Computacional de forma simples e escalável. A Figura 1 apresenta a página inicial da ferramenta.

A Athena dispõe de um editor gráfico que propicia a programação visual dos Sistemas Inteligentes. Esses sistemas podem ser compartilhados com outros pesquisadores para que seja possível discussões sobre as técnicas selecionadas e até mesmo sobre os resultados obtidos. Isso permite a distribuição de conhecimento entre os participantes de um determinado projeto. Além disso, o ambiente encapsula técnicas de IC em módulos, tornando-se desnecessário conhecimento em linguagens de programação para sua utilização.

Com a utilização da ferramenta Athena dentro da sala de aula, os professores passam a ter autonomia para apresentar, durante as aulas, o funcionamento das principais técnicas de Inteligência Computacional e o seu comportamento em diferentes situações. Ela é disponibilizada na Web, permitindo que os usuários acessem o serviço utilizando um navegador conectado à Internet. $\mathrm{O}$ uso da Athena é baseado em selecionar, arrastar

\footnotetext{
${ }^{1}$ Athena WebSite: http://athenasystems.com.br/
} 
V Congresso Brasileiro de Informática na Educação (CBIE 2016)

Anais do XXVII Simpósio Brasileiro de Informática na Educação (SBIE 2016)

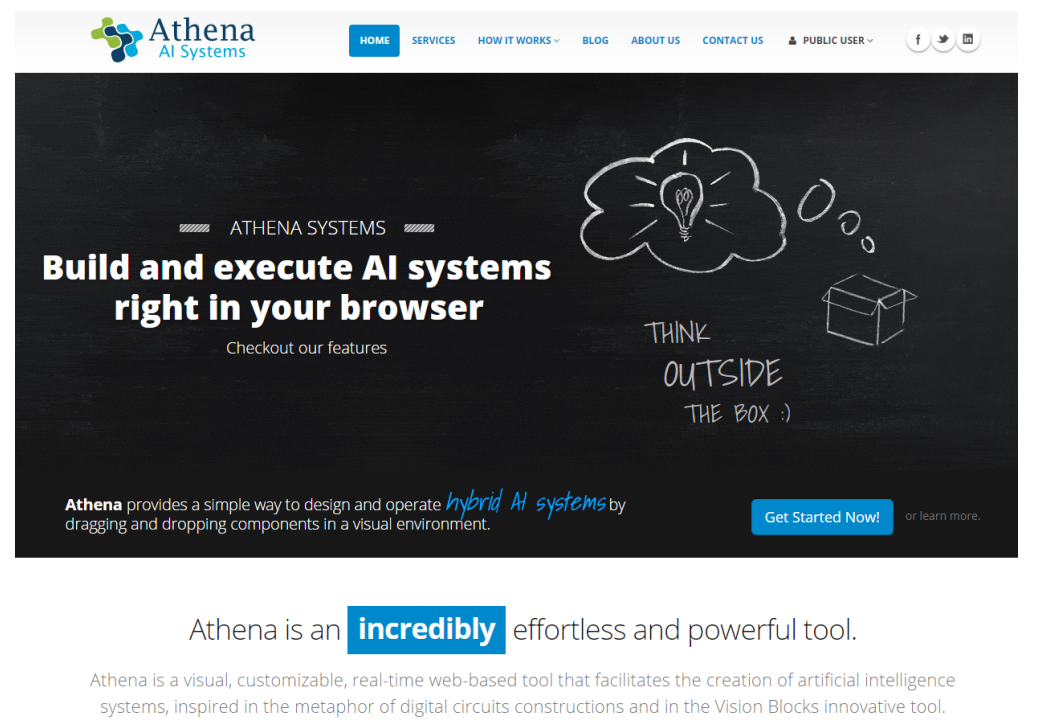

Figura 1. Página inicial da ferramenta Athena.

e conectar diferente componentes visuais (módulos), os quais representam as técnicas de IC disponíveis na ferramenta. Levando-se em conta o atual estado de desenvolvimento, a Athena possui 35 módulos desenvolvidos divididos em cinco categorias:

- Módulos de Inteligência Computacional: esse tipo de módulo encapsula as técnicas de IC, funcionando como motor da ferramenta. Atualmente, a Athena dispõe de pelo menos um módulo para cada subárea da IC, além de alguns módulos relacionados à área de Aprendizagem de Máquina. Dentre os mais importantes dessa categoria, destacam-se: Fuzzy, FCM, gGA, NSGAII, MLP, RBF, AntSystem, MMAS, CLONALG, J48, K-Means, Cobweb;

- Módulos Conversores de Dados: esse tipo de módulo permite a conversão dos dados que trafegam nas ligações do sistema. Além disso, podem ser utilizados para injetar dados nas execuções ou para extrair os resultados em formato de arquivo. Essa categoria contém dois módulos implementados: ReadCSV e WriteCSV, para leitura e escrita em arquivos no formato Comma-separated values(CSV), respectivamente;

- Módulos de Acesso à Base de Dados: esses possibilitam o acesso à bancos de dados externos para extrair entradas para as execuções. Atualmente, três SGBDs são suportados: MySQL, PostgreSQL e Oracle;

- Módulos Auxiliares: essa categoria inclui os módulos que auxiliam a construção dos Sistemas Inteligentes. Por exemplo, módulo de expressões matemáticas para realização de operações entre os dados, módulo estatístico para execução de testes estatísticos, dentre outros;

- Módulos dos Usuários: os usuários da Athena podem submeter novos módulos para a ferramenta, os quais são analisados por uma equipe técnica antes da disponibilização para os demais usuários. Atualmente, essa categoria possui apenas dois módulos: o Textfly - realiza a análise de sentimentos em textos em inglês - e o Twitter - coleta postagens na rede social Twitter.

\subsection{Metodologia da Athena}

O desenvolvimento de Sistemas Inteligentes com a Athena possui cinco etapas: 
V Congresso Brasileiro de Informática na Educação (CBIE 2016)

Anais do XXVII Simpósio Brasileiro de Informática na Educação (SBIE 2016)

1. Informações Básicas: refere-se à criação do sistema de Inteligência Computacional informando nome, descrição e quais usuários poderão acessar suas informações. Essa etapa pode ser visualizada na Figura 2(a);

2. Arquitetura: nessa etapa deve-se selecionar e conectar os módulos com o objetivo de criar uma arquitetura capaz de resolver o problema. O usuário poderá criar diversas arquiteturas com o objetivo de comparar os resultados de diferentes abordagens para resolução do problema-alvo (Figura 2(b));

3. Configuração e Execução: nessa etapa o usuário deve configurar os módulos para habilitar a execução da arquitetura. A Figura 2(c) exibe a tela de configuração da Athena;

4. Análise dos Resultados: ao final do processo de execução, o usuário pode analisar os resultados, que por sua vez serão exibidos por meio de gráficos e tabelas. Além disso, são exibidas informações adicionais coletadas durante a execução dos módulos, por exemplo, número de iterações, tempo de execução, etc. (Figura 2(d));

5. Integração: a ferramenta Athena ainda disponibiliza uma API (Application Programming Interface) de forma a possibilitar diferentes formas de interação com o usuário e com outros sistemas. Essa etapa é opcional.

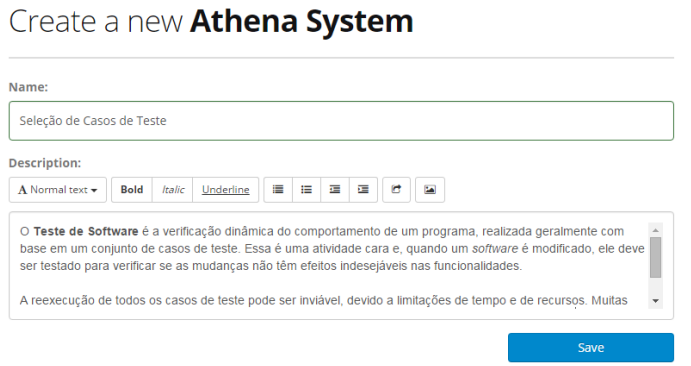

(a) Informações Básicas do Sistema

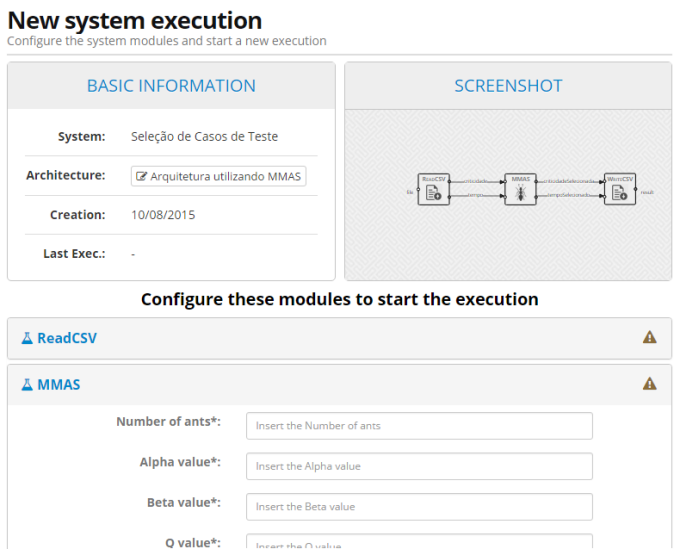

(c) Configuração e Execução

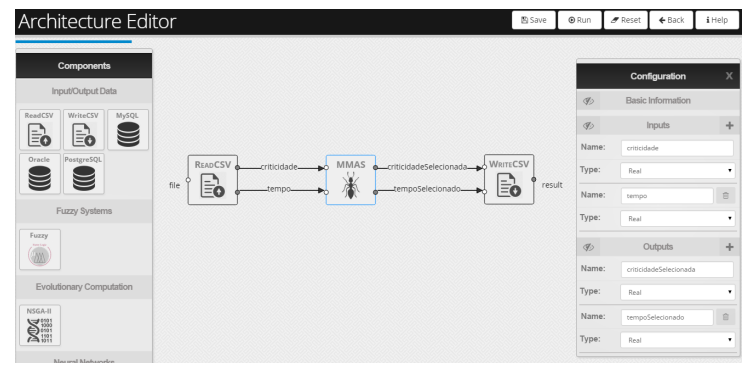

(b) Definição da Arquitetura

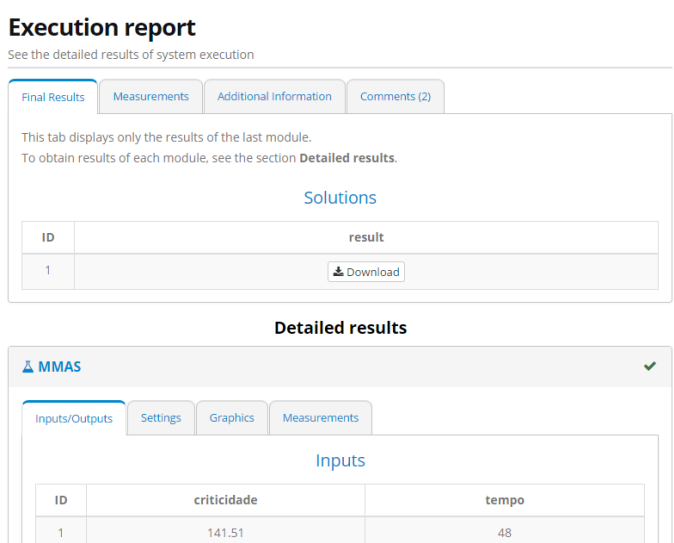

(d) Análise dos Resultados

Figura 2. Etapas de utilização da Athena.

\section{Estudo Experimental}

Após o desenvolvimento da Athena, realizou-se um quasi-experimento controlado [Wohlin et al. 2012]. O propósito desse estudo é analisar o desenvolvimento de Sistemas Inteligentes com e sem a utilização da Athena, no intuito de avaliar o impacto dessas 
V Congresso Brasileiro de Informática na Educação (CBIE 2016)

Anais do XXVII Simpósio Brasileiro de Informática na Educação (SBIE 2016)

duas abordagens, com respeito à eficiência (esforço), eficácia e a percepção de qualidade dos participantes. Esses critérios estão diretamente relacionados ao ensino da IC porque tanto o profissional da educação quanto os alunos desejam mecanismos de aprendizado eficientes, eficazes e que provoquem satisfação ao serem utilizados.

O experimento foi realizado com 15 alunos matriculados na disciplina Tópicos em Inteligência Artificial de um curso de Ciência da Computação, que é ofertada para alunos de graduação e pós-graduação. Dentre os 15 voluntários, havia 4 alunos de mestrado com experiência no mercado de trabalho, 3 alunos de mestrado sem experiência profissional e 8 alunos de graduação. O estudo foi planejado considerando as seguintes questões, métricas e hipóteses:

QP1. O esforço necessário para o desenvolvimento de sistemas de IC com a utilização da Athena é menor que o esforço exigido para desenvolver os mesmos sistemas de IC sem a utilização da Athena? Utilizou-se a medida de tempo em segundos como métrica para essa questão. Hipótese nula, $H_{0}$ esforço: não há diferença no esforço para desenvolver sistemas de IC com e sem o uso da Athena. $H_{0}$ esforço: Tempo (Athena) $=$ Tempo (sem suporte da Athena). Hipótese alternativa, $H_{1}$ esforço: Tempo (Athena) $\neq$ Tempo (sem suporte da Athena).

QP2. A eficácia dos sistemas de IC desenvolvidos com a Athena é maior que a eficácia dos sistemas de IC desenvolvidos sem o apoio da Athena? Utilizou-se como métrica o inverso da quantidade de erros de programação/configuração encontrados nesses sistemas $(1 /($ quantidade_de_erros +1$))$. Hipótese nula, $H_{0}$ eficácia: não há diferença na eficácia dos sistemas de IC desenvolvidos com e sem o uso da Athena. Ho eficácia: Eficácia $($ Athena $)=$ Eficácia (sem suporte da Athena). Hipótese alternativa, $H_{1}$ eficácia: Eficácia (Athena) $\neq$ Eficácia (sem suporte da Athena).

QP3. A percepção de qualidade dos usuários quanto ao desenvolvimento de sistemas de IC com o auxílio da Athena é maior que sistemas de IC desenvolvidos sem o suporte dessa ferramenta? Devido a natureza subjetiva dessa questão, foram utilizados questionários para avaliar a satisfação dos voluntários. Hipótese nula, $H_{0}$ qualidade: a qualidade dos sistemas de IC criados utilizando a Athena é igual à percepção de qualidade de sistemas criados sem o suporte da ferramenta. $H_{0}$ qualidade: Qualidade (Athena) = Qualidade (sem suporte da Athena). Hipótese alternativa, $H_{1}$ qualidade: Qualidade (Athena) $\neq$ Qualidade (sem suporte da Athena).

Tabela 1. Desenho experimental utilizado na avaliação empírica da Athena.

\begin{tabular}{|c|c|c|c|c|}
\cline { 2 - 5 } \multicolumn{1}{c|}{} & \multicolumn{3}{c|}{ Desenvolvimento de Sistemas de IC } \\
\cline { 2 - 5 } \multicolumn{1}{c|}{$\mathbf{1}^{\mathbf{a}}$ Etapa } & \multicolumn{2}{c|}{$\mathbf{2}^{\mathrm{a}}$ Etapa } \\
\cline { 2 - 5 } Grupos & $1^{\mathrm{a}}$ Sessão & $2^{\mathrm{a}}$ Sessão & $3^{\mathrm{a}}$ Sessão & $4^{\mathrm{a}}$ Sessão \\
\hline \multirow{2}{*}{ Grupo Único } & \multicolumn{2}{c|}{ Abordagem com Athena } & \multicolumn{2}{c|}{ Abordagem Manual } \\
& $\begin{array}{c}\text { Treinamento } \\
(2 \mathrm{~h})\end{array}$ & $\begin{array}{c}\text { Não-Híbrido } \\
\text { Híbrido }\end{array}$ & $\begin{array}{c}\text { Treinamento } \\
(2 \mathrm{~h})\end{array}$ & $\begin{array}{c}\text { Não-Híbrido } \\
\text { Híbrido }\end{array}$ \\
\hline
\end{tabular}

Após a construção das questões, definiu-se o desenho experimental, ou seja, o modo como as avaliações foram realizadas (Tabela 1). $\mathrm{O}$ estudo foi executado em duas etapas e quatro sessões. Na primeira etapa, todos os participantes desenvolveram um sistema não-híbrido para solucionar o problema da estimação do tamanho de nanopartículas de prata (Estimação do Tamanho de AgNP) [de Aragão et al. 2015] e outro híbrido (que 
V Congresso Brasileiro de Informática na Educação (CBIE 2016)

Anais do XXVII Simpósio Brasileiro de Informática na Educação (SBIE 2016)

integra diferentes técnicas de IC) para solucionar o problema da alocação de equipes em projetos de desenvolvimento de software (Alocação de Equipes) [Britto et al. 2012] com o auxílio da Athena. Na segunda etapa, eles desenvolveram os mesmos sistemas sem o apoio da Athena, ou seja, utilizando frameworks conhecidos na área (JFuzzyLogic, Neuroph Studio e JMetal). Antes do início de cada etapa, os participantes receberam um treinamento de 2 horas sobre as técnicas e ferramentas de IC usadas no experimento.

\subsection{Resultados e Discussões}

A interpretação dos dados coletados iniciou-se a partir da construção de box-plots (Figura 3) para que fosse possível visualizar a dispersão e variação dos dados, a assimetria da distribuição, os quartis e os outliers (medidas discrepantes).

Analisando a Figura 3(a), pode-se perceber que o desenvolvimento dos Sistemas Inteligentes demandaram menos esforço utilizando a Athena, esse resultado já era esperado devido ao fato da ferramenta remover a complexidade de implementação das técnicas de IC. Isso fica mais evidente no desenvolvimento do sistema para o problema Alocação de Equipes que necessita da construção de um sistema híbrido. Em relação à eficácia, a Figura 3(b) mostra que a implementação dos sistemas utilizando a Athena gerou menos erros. Além disso, verificou-se que tanto para eficácia quanto para o esforço, a variabilidade dos dados foi menor utilizando a Athena.
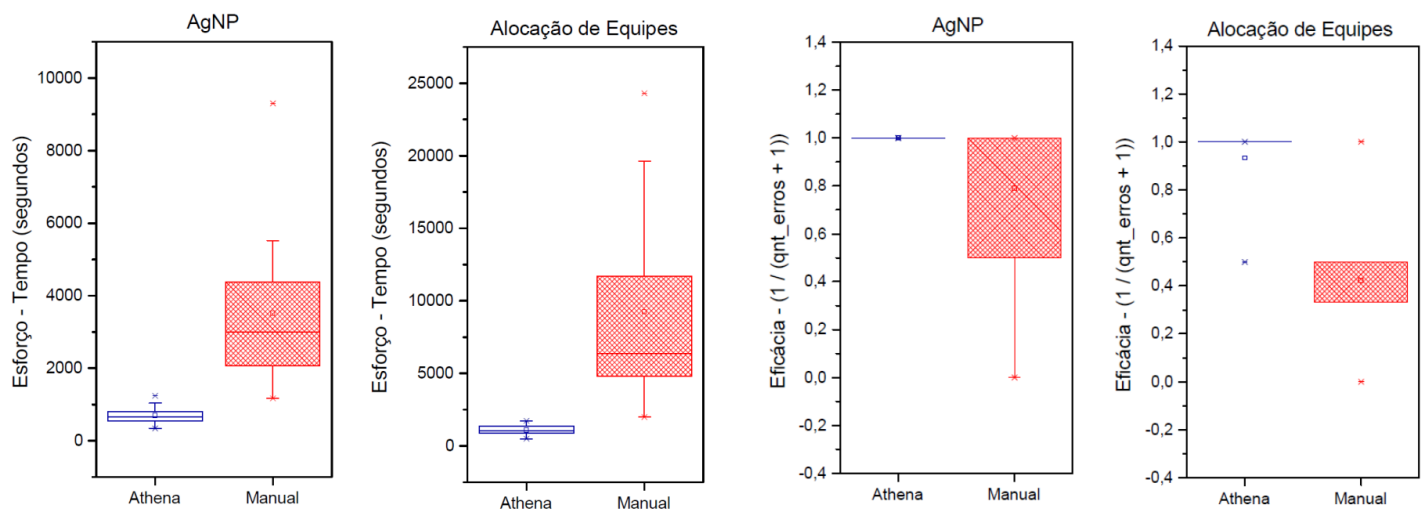

(a) Box-plot para os dados de esforço.

(b) Box-plot para os dados de eficácia.

Figura 3. Box-plots dos resultados do experimento.

Após a análise dos box-plots foram realizados alguns testes estatísticos para verificar se os dados obtidos com auxilio da Athena são, de fato, estatisticamente diferentes dos dados obtidos sem a ferramenta. Inicialmente, os dados foram submetidos ao teste de normalidade Anderson-Darling. Os resultados do teste indicaram que, com nível de confiança de 95\%, os conjuntos de dados não seguem a distribuição normal. Dessa forma, utilizou-se o teste não-paramétrico de Wilcoxon com amostras pareadas para avaliar as hipóteses do experimento.

O teste Wilcoxon retornou um $p$-value igual a $6 \times 10^{-5}$ quando aplicado aos dados de esforço coletados durante o estudo. Dessa forma, pode-se afirmar com $99 \%$ de certeza (nível de significância igual a 0,01 ) que as duas distribuições são estatisticamente diferentes. Portanto, a hipótese $H_{0}$ esforço foi rejeitada e a resposta à Questão de Pesquisa 1 (QP1) é: o esforço necessário para o desenvolvimento de sistemas de IC com a 
V Congresso Brasileiro de Informática na Educação (CBIE 2016)

Anais do XXVII Simpósio Brasileiro de Informática na Educação (SBIE 2016)

utilização da ferramenta Athena é, de fato, menor que o esforço exigido para desenvolver os mesmos sistemas de IC sem o apoio da ferramenta.

As amostras de eficácia dos sistemas criados com e sem o apoio da Athena para solucionar o problema Estimação do Tamanho de AgNP não são estatisticamente diferentes ( $p$-value igual a 0,125 e nível de significância igual a 0,01). Entretanto, as amostras obtidas para o problema Alocação de Equipes são estatisticamente diferentes ( $p$-value igual a $4,9 \times 10^{-4}$ e nível de significância igual a 0,01 ). Essa distinção deve-se ao fato de que a resolução do problema Alocação de Equipes envolve mais de uma técnica de IC. Isso aumenta a complexidade do desenvolvimento e acaba gerando mais erros. Portanto, a hipótese $H_{0}$ eficácia não pode ser rejeitada e a resposta à Questão de Pesquisa 2 (QP2) é: a eficácia dos sistemas de IC desenvolvidos com a Athena não é maior que a eficácia dos sistemas de IC desenvolvidos sem o apoio da ferramenta. Entretanto, observou-se que a construção de soluções híbridas sem o auxílio da Athena é propensa a erros.

Realizou-se a análise do questionário pós-experimento para verificar a percepçãode qualidade dos participantes envolvidos no estudo. A maioria (80\%) respondeu que o estudo foi bem executado e que os treinamentos foram adequados ou muito adequados ao propósito do experimento. Quanto à Athena, $80 \%$ dos voluntários qualificaram a Athena como excelente e todos (100\%) atestaram que estão dispostos a utilizar a ferramenta em ambiente acadêmico e industrial. Desse modo, a hipótese $H_{0}$ qualidade foi rejeitada pelo pesquisador e a resposta à Questão de Pesquisa 3 (QP3) é: considerando o contexto deste estudo e os comentários dos participantes, a percepção de qualidade dos usuários quanto ao desenvolvimento de Sistemas de IC com o auxílio da Athena é maior que sistemas de IC desenvolvidos sem o suporte dessa ferramenta.

Esses resultados fundamentam a proposta deste trabalho de que a Athena pode ser utilizada por educadores e alunos no ensino e na aprendizagem da IC. Isso deve-se ao fato de que a ferramenta reduz o esforço gasto na construção dos Sistemas Inteligentes, permitindo ao professor demonstrar o funcionamento das técnicas dentro de sala de aula e possibilitando ao aluno uma referência em trabalhos que exijam a implementação manual das técnicas. Quanto à qualidade, a Athena pode reduzir a quantidade de erros presentes nos Sistemas Inteligentes, fornecendo resultados mais confiáveis. Finalmente, devido a boa usabilidade da ferramenta, estudar o funcionamento das técnicas de IC se torna bem mais atrativo, principalmente para alunos de áreas que não estão diretamente ligadas à Ciência da Computação.

\subsection{Ameaças à Validade}

Segundo Wohlin, existem alguns tipos de ameaças à validade dos resultados de um estudo experimental, portanto identificou-se alguns desses fatores com o objetivo de mitigar as suas influências nos resultados desse experimento [Wohlin et al. 2012].

A primeira categoria de ameaças preocupa-se com a necessidade de assegurar que, caso haja uma relação entre tratamento e os resultados, essa relação seja do tipo causaefeito e que não seja decorrente de um fator que não foi controlado. Essa categoria, intitulada ameaças à validade interna, inclui questões como a história, maturação e ameaças sociais. Os resultados do estudo podem ser enviesados pela história, caso o estudo ocorra logo após a final de um grande campeonato de futebol, por exemplo. Para evitar isso, a data foi escolhida cuidadosamente. 
V Congresso Brasileiro de Informática na Educação (CBIE 2016)

Anais do XXVII Simpósio Brasileiro de Informática na Educação (SBIE 2016)

Outro efeito que pode alterar os resultados do estudo é a maturação, pois os participantes podem ser afetados negativamente por tarefas chatas ou positivamente com o aprendizado durante o experimento. Para evitar os efeitos da maturação, a Athena foi utilizada primeiro pelos participantes. Assim, a possibilidade de que o aprendizado favorecesse a ferramenta foi descartada. Por fim, os participantes podem pensar que estão dentro de uma competição e isso também pode alterar os resultados. Esse é um tipo de ameaça social que foi combatida com uma apresentação dos objetivos do experimento, além de deixar claro que eles não estavam inseridos em nenhuma competição.

A categoria de ameaças à validade externa preocupa-se com a generalização dos resultados obtidos pelo estudo. Existem dois riscos principais: utilizar participantes não adequados e realizar o experimento em ambiente não adequado ou com ferramentas inadequadas. Para mitigar esses riscos, a seleção dos participantes contou com alunos de pós-graduação e graduação e as ferramentas escolhidas foram selecionadas de acordo com estudos que comprovam sua utilização na prática industrial e acadêmica. Entretanto, há um ponto que precisa ser discutido: o experimento avaliou de forma indireta o impacto da Athena no ensino de IC. Isso porque o estudo focou no esforço, eficácia e percepção de qualidade no desenvolvimento de Sistemas Inteligentes. Dessa forma, pretende-se abordar em trabalhos futuros uma avaliação diretamente relacionada ao ensino.

\section{Conclusão}

O estudo dos Sistemas Inteligentes vem se mostrando cada vez mais importante devido à sua capacidade de solucionar diversos tipos de problemas nas mais variadas áreas do conhecimento. Isso reforça a transversalidade dessa linha de pesquisa e justifica não só o crescimento do número de trabalhos científicos que aplicam técnicas inteligentes em ambientes complexos e dinâmicos mas, também, o fortalecimento do desenvolvimento de ferramentas aptas a auxiliar o ensino e a construção de Sistemas Baseados em IC.

A Athena foi desenvolvida inicialmente para apoiar a construção de Sistemas Inteligentes, entretanto, após sua utilização por professores de determinados grupos de pesquisa, percebeu-se que ela também poderia ser utilizada no ensino de IC. Para verificar a sua adequação a esse propósito, realizou-se um experimento com alunos do curso de Ciência da Computação em nível de graduação e pós-graduação. Os resultados indicam que a Athena pode ser útil para educadores e alunos envolvidos com o ensino e aprendizagem de IC. Portanto, com base nos resultados experimentais obtidos, acredita-se que os objetivos principais (apoiar o ensino de IC e facilitar a construção de Sistemas Inteligentes) propostos para o desenvolvimento da Athena foram alcançados.

As principais contribuições deste trabalho são: i) a apresentação de uma ferramenta visual para apoiar o ensino da IC descrevendo suas principais funcionalidades; ii) a descrição de um estudo experimental realizado em ambiente controlado com o objetivo de avaliar o desempenho de alunos utilizando a ferramenta na construção de Sistemas Inteligentes para a resolução de problemas reais.

Destaca-se como trabalhos futuros, a replicação do estudo experimental com mais alunos e profissionais que atuem com IC tanto no ensino como na prática industrial, além de voluntários de outras áreas do conhecimento. 
V Congresso Brasileiro de Informática na Educação (CBIE 2016)

Anais do XXVII Simpósio Brasileiro de Informática na Educação (SBIE 2016)

\section{Referências}

Amato, F., Lopez, A., Peña-Méndez, E. M., Vaňhara, P., Hampl, A., and Havel, J. (2013). Artificial neural networks in medical diagnosis. Journal of applied biomedicine, 11(2):47-58.

Azevedo, B. F. and Tavares, O. L. (1998). Um sistema tutor inteligente para suporte à aprendizagem de 'conceitos de orientação à objetos. Revista Engenharia, UFES, Espírito Santo, Novembro.

Braga, R., Oliveira, P. A., Souza, M., Neto, P. S., Rabêlo, R., and Britto, R. (2015). Ferramentas para desenvolvimento de sistemas baseados em inteligência computacional: Um mapeamento sistemático. In Anais do Simpósio Brasileiro de Automação Inteligente, volume 12 .

Britto, R., Neto, P. S., Rabelo, R., Ayala, W., and Soares, T. (2012). A hybrid approach to solve the agile team allocation problem. In IEEE Congress on Evolutionary Computation (CEC), 2012, Brisbane, pages 1-8.

de Aragão, A. P., da Silva, D. A., Rabêlo, R. d. A. L., de Oliveira, T. M., Ulissses, P. H. D. C., and Fernandes, R. A. S. (2015). Uma aplicação de redes neurais artificiais e otimização por enxame de partículas para estimativa do tamanho de nanopartículas de prata. In Anais do Simposio Brasileiro de Automação Inteligente, volume 12.

Diekmann, A. and Gutjahr, S. (1998). Prediction of the euro-dollar future using neural networks a case study for financial time series prediction. In Proceedings of the International Symposium on Intelligent Data Engineering and Learning, volume 98, pages 1998-13.

Engelbrecht, A. P. (2007). Computational Intelligence: An Introduction. Wiley Publishing, 2nd edition.

Laroza, J. and Seabra, R. (2015). Rea-uml: Recurso educacional aberto para ensino da uml. In Anais do Simpósio Brasileiro de Informática na Educação, volume 26, page 11.

Monteiro, B., Oliveira, E., Gomes, A. S., and Neto, F. M. M. (2015). Youubi: Ambiente de aprendizagem ubíqua. In Anais dos Workshops do Congresso Brasileiro de Informática na Educação, volume 4, page 1.

Oliveira, P., Souza, M., Braga, R., Brito, R., Lira Rabelo, R., and Neto, P. (2014). Athena: A visual tool to support the development of computational intelligence systems. In IEEE 26th International Conference on Tools with Artificial Intelligence (ICTAI), 2014, pages 950-959.

Santos-Neto, P., Britto, R., Soares, T., Ayala, W., Cruz, J., and Rabelo, R. (2012). Regression testing prioritization based on fuzzy inference systems. In SEKE, pages 273-278. Knowledge Systems Institute Graduate School.

Wohlin, C., Runeson, P., Höst, M., Ohlsson, M. C., Regnell, B., and Wesslén, A. (2012). Experimentation in software engineering. Springer.

Zhu, T., Tso, S., and Lo, K. (2004). Wavelet-based fuzzy reasoning approach to powerquality disturbance recognition. IEEE Transactions on Power Delivery. 